\title{
Trends in the elastic response of binary early transition metal nitrides
}

\author{
David Holec, ${ }^{1, *}$ Martin Friák, ${ }^{2}$ Jörg Neugebauer, ${ }^{2}$ and Paul H. Mayrhofer ${ }^{1}$ \\ ${ }^{1}$ Department of Physical Metallurgy and Materials Testing, Montanuniversität Leoben, Franz-Josef-Strasse 18, AT-8700 Leoben, Austria \\ ${ }^{2}$ Max-Planck-Institut für Eisenforschung GmbH, Max-Planck-Strasse 1, DE-40237 Düsseldorf, Germany
}

(Received 15 November 2011; published 2 February 2012)

\begin{abstract}
Motivated by an increasing demand for coherent data that can be used for selecting materials with properties tailored for specific application requirements, we studied elastic response of nine binary early transition metal nitrides ( $\mathrm{ScN}$, TiN, VN, YN, ZrN, NbN, LaN, HfN, and TaN) and AlN. In particular, single-crystal elastic constants, Young's modulus in different crystallographic directions, polycrystalline values of shear and Young's moduli, and the elastic anisotropy factor were calculated. Additionally, we provide estimates of the third order elastic constants for the ten binary nitrides.
\end{abstract}

DOI: 10.1103/PhysRevB.85.064101

PACS number(s): 62.20.-x, 31.15.A-, 81.05.Je, 62.20.de

\section{INTRODUCTION}

Nitride compounds are a prominent class of materials with applications spanning from protective hard coatings (mostly transition metal nitrides, TMNs, of the IIIB-VIB group but also, e.g., BN or SiN), ${ }^{1,2}$ to optoelectronic devices (mostly IIIA and VA groups, but also, e.g., $\mathrm{ScN}$ or TiN) ${ }^{3}$, to potential hydrogen storage materials such as $\mathrm{Li}_{3} \mathrm{~N}^{4}$

When it comes to their superior mechanical properties such as high hardness and Young's modulus, TMNs, and in particular TiN are often the industrial material of choice for surface coating of, e.g., cutting tools. The properties of simple binary compounds can be successfully enhanced by forming metastable alloys, e.g, $\mathrm{Ti}_{1-x} \mathrm{Al}_{x} \mathrm{~N}^{1}$, which at higher temperatures age hardens before decomposing into its stable constituents. ${ }^{5}$ Recent studies have shown beneficial effects (such as increased oxidation resistance or retardation of the final decomposition step to higher temperatures) of additional alloying elements in $\mathrm{Ti}_{1-x} \mathrm{Al}_{x} \mathrm{~N}^{5-8}$ and other systems. ${ }^{9}$ Another approach how to improve material properties is via multilayer design, where individual layers are typically simple binary or ternary systems. ${ }^{10-12}$

A modern way of designing new and improving current materials is to combine experiment with modeling. For simple and/or small systems, quantum mechanical first-principles approaches can be used. However, these are typically limited to several hundreds of atoms and, for example, multilayers, crack propagation, or nanoindentor tip-layer contact become difficult topics to handle. Here, the continuum mechanics employing finite element method (FEM) proves to be a successful tool. ${ }^{13-15}$ A key prerequisite to perform FEM calculations is the knowledge of the elastic properties of the studied materials, which are not always experimentally available (e.g., because some phases are stable only in the multilayer arrangement, but not as a bulk material). In such cases, the elastic constants can be provided by the first-principles calculations.

The literature on the first-principles calculations of early TMNs (group IIIB-VB) is vast. The main focus of those

Published by the American Physical Society under the terms of the Creative Commons Attribution 3.0 License. Further distribution of this work must maintain attribution to the author(s) and the published article's title, journal citation, and DOI. papers is the electronic structure and related material chemistry problems, while the calculation of the elastic properties is often only a minor part of the results. Additionally, a lot of those reports focus only on one or a few systems. There are some exhaustive reports on the chemical trends in the early TMNs, ${ }^{16-21}$ but apart from Refs. 20 and 21 they do not discuss the elastic properties. In addition, there are some discrepancies between reported values (e.g., $C_{11}$ for $\mathrm{ZrN}$ between $304 \mathrm{GPa}^{22}$ and $616 \mathrm{GPa}^{20}$ ), which are worth cross checking.

The aim of the present paper is to give a comprehensive overview on elastic properties of early transition-metal nitrides and AlN, since these materials are or have the potential to be used as protective coatings. ${ }^{23}$ In particular, we investigate ten binary systems: AlN, ScN, TiN, VN, YN, $\mathrm{ZrN}, \mathrm{NbN}$, $\mathrm{LaN}, \mathrm{HfN}$, and TaN. We focus on the cubic variant (B1, $F m \overline{3} m, \mathrm{NaCl}$ prototype), which is the stable configuration of all of them apart from $\mathrm{AlN}, \mathrm{NbN}$, and $\mathrm{TaN}$ being metastable in this configuration. The single-crystal elastic constants are validated by several independent approaches as well as by a comparison with available theoretical and experimental data. Subsequently, we calculate directionally resolved Young's modulus, anisotropy factors, and polycrystalline elastic properties of these compounds, and rationalize the trends in terms of their electronic structure and bonding.

\section{METHODOLOGY}

\section{A. Deformation modes}

The linear elastic response of cubic materials is fully described by three independent components $c_{x x x x}, c_{x x y y}$, and $c_{x y x y}$ of the fourth rank tensor of the second-order elastic constants (SOECs). It is convenient to represent this tensor with a $6 \times 6$ matrix:

$$
C_{i j}=\left(\begin{array}{cccccc}
C_{11} & C_{12} & C_{12} & 0 & 0 & 0 \\
C_{12} & C_{11} & C_{12} & 0 & 0 & 0 \\
C_{12} & C_{12} & C_{11} & 0 & 0 & 0 \\
0 & 0 & 0 & C_{44} & 0 & 0 \\
0 & 0 & 0 & 0 & C_{44} & 0 \\
0 & 0 & 0 & 0 & 0 & C_{44}
\end{array}\right),
$$

where $C_{11}=c_{x x x x}, C_{12}=c_{x x y y}$, and $C_{44}=C_{66}=c_{x y x y}$. Here, we make use of the Voigt notation $x x \sim 1, y y \sim 2$, 
$z z \sim 3, y z \sim 4, x z \sim 5$, and $x y \sim 6$. An additional relationship links SOECs with the bulk modulus $B$,

$$
B=\frac{1}{3}\left(C_{11}+2 C_{12}\right) .
$$

$B$ describes the elastic response of materials to volume changes, and it is obtained as a fitting parameter from the Birch-Murnaghan equation of state. ${ }^{24}$ Consequently, two other deformation modes are needed to obtain all independent components of the cubic elastic tensor.

The first pair consists of orthorhombic and monoclinic deformations. The orthorhombic mode results in a strain tensor,

$$
\varepsilon_{\text {orth }}(\delta)=\left(\begin{array}{ccc}
\delta & 0 & 0 \\
0 & -\delta & 0 \\
0 & 0 & \frac{\delta^{2}}{1-\delta^{2}}
\end{array}\right),
$$

and the corresponding strain energy density $U(\delta)$ is

$$
U_{\text {orth }}(\delta)=\mathcal{E}_{\text {tot }}(\delta)-\mathcal{E}_{\text {eq }}=\left(C_{11}-C_{12}\right) \delta^{2}+\mathcal{O}\left(\delta^{3}\right) .
$$

Here, $\mathcal{E}_{\text {tot }}(\delta)$ and $\mathcal{E}_{\text {eq }}$ are the total energies per unit volume, corresponding to $\varepsilon_{\text {orth }}(\delta)$ and $\varepsilon_{\text {orth }}(0)$, respectively. A monoclinic deformation yielding a strain tensor

$$
\varepsilon_{\mathrm{mon}}(\delta)=\left(\begin{array}{ccc}
0 & \frac{1}{2} \delta & 0 \\
\frac{1}{2} \delta & 0 & 0 \\
0 & 0 & \frac{\delta^{2}}{4-\delta^{2}}
\end{array}\right)
$$

is used to evaluate the $C_{44}$ elastic constant from the corresponding strain energy density

$$
U_{\text {mon }}(\delta)=\mathcal{E}_{\text {tot }}(\delta)-\mathcal{E}_{\text {eq }}=\frac{1}{2} C_{44} \delta^{2}+\mathcal{O}\left(\delta^{3}\right) .
$$

One should note that these two modes keep the unit cell volume constant.

The second pair of deformations, which is also often used, is a pair of tetragonal and triclinic distortion. The tetragonal deformation corresponds to a strain matrix

$$
\varepsilon_{\text {tet }}(\delta)=\left(\begin{array}{ccc}
-\frac{1}{2} \delta & 0 & 0 \\
0 & -\frac{1}{2} \delta & 0 \\
0 & 0 & \delta
\end{array}\right)
$$

producing a strain energy density

$$
U_{\text {tet }}(\delta)=\mathcal{E}_{\text {tot }}(\delta)-\mathcal{E}_{\text {eq }}=\frac{3}{4}\left(C_{11}-C_{12}\right) \delta^{2}+\mathcal{O}\left(\delta^{3}\right) .
$$

The $C_{44}$ elastic constants is obtained from a trigonal distortion with a strain tensor

$$
\varepsilon_{\mathrm{tri}}(\delta)=\left(\begin{array}{ccc}
0 & \delta & 0 \\
\delta & 0 & 0 \\
0 & 0 & 0
\end{array}\right)
$$

and a strain energy density

$$
U_{\text {tri }}(\delta)=\mathcal{E}_{\text {tot }}(\delta)-\mathcal{E}_{\text {eq }}=2 C_{44} \delta^{2}+\mathcal{O}\left(\delta^{3}\right) .
$$

These two deformations are volume nonconserving.

Recently, Zhao et al. ${ }^{25}$ and Łopuszyński and Majewski ${ }^{26}$ proposed a set of six deformation matrices that allow for estimation of second- and third-order ${ }^{27}$ elastic constants of
TABLE I. Coefficients from Eq. (12) for various deformation matrices $A 1-A 6$.

\begin{tabular}{lll}
\hline \hline & \multicolumn{1}{c}{$\mathcal{A}$} & \multicolumn{1}{c}{$\mathcal{B}$} \\
\hline$A 1$ & $\frac{1}{2} C_{11}$ & $\frac{1}{6} C_{111}$ \\
$A 2$ & $C_{11}+C_{12}$ & $\frac{1}{3} C_{111}+C_{112}$ \\
$A 3$ & $\frac{3}{2} C_{11}+3 C_{12}$ & $\frac{1}{2} C_{111}+3 C_{112}+C_{123}$ \\
$A 4$ & $\frac{1}{2} C_{11}+2 C_{44}$ & $\frac{1}{6} C_{111}+2 C_{144}$ \\
$A 5$ & $\frac{1}{2} C_{11}+2 C_{44}$ & $\frac{1}{6} C_{111}+2 C_{166}$ \\
$A 6$ & $6 C_{44}$ & $8 C_{456}$ \\
\hline \hline
\end{tabular}

cubic materials at the same time. These are

$$
\begin{aligned}
A 1 & =\left(\begin{array}{lll}
\delta & 0 & 0 \\
0 & 0 & 0 \\
0 & 0 & 0
\end{array}\right), & A 2 & =\left(\begin{array}{lll}
\delta & 0 & 0 \\
0 & \delta & 0 \\
0 & 0 & 0
\end{array}\right), \\
A 3 & =\left(\begin{array}{lll}
\delta & 0 & 0 \\
0 & \delta & 0 \\
0 & 0 & \delta
\end{array}\right), & A 4 & =\left(\begin{array}{lll}
\delta & 0 & 0 \\
0 & 0 & \delta \\
0 & \delta & 0
\end{array}\right), \\
A 5 & =\left(\begin{array}{lll}
\delta & \delta & 0 \\
\delta & 0 & 0 \\
0 & 0 & 0
\end{array}\right), & A 6 & =\left(\begin{array}{lll}
0 & \delta & \delta \\
\delta & 0 & \delta \\
\delta & \delta & 0
\end{array}\right) .
\end{aligned}
$$

The strain energy density in these cases is

$$
U_{A}(\delta)=\mathcal{E}_{\text {tot }}(\delta)-\mathcal{E}_{\text {eq }}=\mathcal{A} \delta^{2}+\mathcal{B} \delta^{3}+\mathcal{O}\left(\delta^{4}\right),
$$

where the coefficients $\mathcal{A}$ and $\mathcal{B}$ are specific combinations of $C_{i j}$ and $C_{i j k}$ as given in Table I.

\section{B. Calculation details}

Quantum-mechanical calculations employing density functional theory (DFT) ${ }^{28,29}$ were carried out using Vienna $a b$ initio simulation package. ${ }^{30,31}$ Projector augmented-wave pseudopotentials ${ }^{32}$ together with the generalized gradient approximation (GGA), as parametrized by Wang and Perdew, ${ }^{33}$ for the exchange and correlation potential were used. The plane-wave cutoff energies and the $\boldsymbol{k}$-vector samplings of the Brillouin zone were carefully checked to provide a total energy accuracy in the order of $1 \mathrm{meV} /$ at or better. They are listed in Table II together with the used pseudopotentials; the suffices _sv and _pv refer to the exact valence configuration taking into account explicitly also the $s$ and $p$ closed-shell electrons, respectively.

\section{RESULTS AND DISCUSSION}

\section{A. Equilibrium properties}

The optimized lattice constants $a$, formation energies $E_{f}$, and mass densities $\rho$ are summarized in Table III. Since there has been a vast number of publications on experimental as well as calculated equilibrium structure parameters of these early TMN compounds (see, e.g., Refs. 16,18,19,21,34 and 35, 
TABLE II. An overview of PAW-GGA pseudopotentials, planewave cutoff energies, and the Monkhorst-Pack sampling of the Brillouin zone used in this study.

\begin{tabular}{lcc}
\hline \hline pseudopotential & $E_{\text {cut }}(\mathrm{eV})$ & $\boldsymbol{k}$-point sampling \\
\hline Al & 800 & $5 \times 5 \times 5$ \\
Sc_sv & 800 & $7 \times 7 \times 7$ \\
Ti_pv & 400 & $17 \times 17 \times 17$ \\
V_pv & 800 & $11 \times 11 \times 11$ \\
Y_sv & 800 & $7 \times 7 \times 7$ \\
Zr_sv & 700 & $13 \times 13 \times 13$ \\
Nb_sv & 600 & $15 \times 15 \times 15$ \\
La & 800 & $7 \times 7 \times 7$ \\
Hf_pv & 700 & $11 \times 11 \times 11$ \\
Ta_pv & 600 & $15 \times 15 \times 15$ \\
\hline \hline
\end{tabular}

and references therein), we limit the comparison of the here calculated lattice parameters to the experimental data from Ref. 36. The calculated lattice constants (with the exception of $\mathrm{VN}$ ) are, as expected for GGA, slightly larger than the experimental values. The error is smaller then $1 \%$ except for the case of $\mathrm{TaN}$, where a deviation of about $1.5 \%$ is obtained. This is likely to be related to the fact that cubic $\mathrm{TaN}$ is metastable and prefers $\mathrm{N}$-deficient configurations resulting in a significant decrease of the lattice parameter with respect to a stoichiometric configuration. ${ }^{37}$

The trends in the energy of formation, i.e., less negative values as one moves from the IIIB to the VB group, as well as the absolute numbers agree well with those presented by Rovere et al. ${ }^{35}$ Formation energy was calculated as a difference between the total energy of the binary nitride, and the total energy of the respective metal in its stable crystalline form and the molecule of nitrogen.

Lastly, from the calculated lattice parameters (equilibrium volume) and the atomic weights we computed mass densities. Again, apart from the TaN case, where the under-stoichiometry of the experimental compound is likely to play a role, the agreement with experimental values is satisfactory.

TABLE III. Calculated lattice constants $a$, formation energy $E_{f}$, and mass density $\rho$. The experimental lattice constants $a_{\text {exp }}$ are taken from Ref. 36, the experimental values of density $\rho_{\exp }$ are from Ref. 38, and those marked with an asterisks are from Ref. 39.

\begin{tabular}{lccccc}
\hline \hline & $a(\AA)$ & $a_{\exp }(\AA)$ & $E_{f}(\mathrm{eV} / \mathrm{at})$ & $\rho\left(\mathrm{g} / \mathrm{cm}^{3}\right)$ & $\rho_{\exp }\left(\mathrm{g} / \mathrm{cm}^{3}\right)$ \\
\hline $\mathrm{AlN}$ & 4.069 & 4.045 & -2.285 & 4.04 & \\
$\mathrm{ScN}$ & 4.516 & 4.440 & -2.958 & 4.25 & \\
$\mathrm{TiN}$ & 4.253 & 4.241 & -2.752 & 5.34 & $5.40^{\star}$ \\
$\mathrm{VN}$ & 4.127 & 4.139 & -1.998 & 6.14 & $6.13,6.0^{\star}$ \\
$\mathrm{YN}$ & 4.917 & 4.894 & -2.737 & 5.75 & \\
$\mathrm{ZrN}$ & 4.618 & 4.578 & -2.716 & 7.10 & $7.32^{\star}$ \\
$\mathrm{NbN}$ & 4.427 & 4.389 & -2.001 & 8.19 & $8.47,7.3^{\star}$ \\
$\mathrm{LaN}$ & 5.306 & 5.293 & -2.350 & 6.80 & 6.73 \\
$\mathrm{HfN}$ & 4.538 & 4.525 & -2.783 & 13.68 & $13.80,13.8^{\star}$ \\
$\mathrm{TaN}$ & 4.426 & 4.358 & -1.869 & 14.94 & $13.70,14.3^{\star}$ \\
\hline \hline
\end{tabular}

\section{B. Single-crystal elastic constants}

When calculating the single-crystal elastic constants as described in Sec. II A, one should check how the fitted $C_{i j} \mathrm{~s}$ depend on the maximum deformation, $\delta_{\max }$, i.e., on the range of deformations applied to the unit cell. When $\delta_{\max }$ is too small, the accuracy of $C_{i j}$ s is likely to be influenced by the numerical inaccuracies of the DFT calculations, while nonlinear elastic (and perhaps also plastic) effects are no longer negligible for too large $\delta_{\max } \cdot{ }^{25}$ To illustrate this behavior, we plot in Fig. 1 the $C_{11}$ and $C_{44}$ elastic constants of $\mathrm{ZrN}$ as a function of $\delta_{\max }$ and the order of the fitting polynomial. It follows, that with increasing order of the fitting polynomial, the plateau region where the specific elastic constant is independent of $\delta_{\max }$, enlarges. At the same time, the onset of the plateau shifts to higher values $\delta_{\max }$. The reason is that a high fitting order leads to an overfitting of the too few data points for a small $\delta_{\max }$. In extreme cases, such overfitting may lead to incorrect plateaus, as shown, e.g., for $C_{44}$ (monoclinic deformation) using a $13^{\text {th }}$ order fitting polynomial.

In general, the combination of tetragonal and trigonal deformations gives more robust results for the early TMN in the cubic structure then the combination of orthorhombic and monoclinic deformations. Nevertheless, the most robust results in terms of plateau values scatter and the dependence on the order of the fitting polynomial, were obtained when employing the deformation matrices $A 1$ and $A 6$ (see Fig. 1).

Recently, Udyansky et al. ${ }^{40}$ showed that the elastic constants of $\alpha$-Fe are also highly sensitive to the value of the smearing parameter $\sigma$ in the Methfessel-Paxton scheme. We have therefore checked the convergence of $C_{i j}$ s also with respect to $\sigma$. It turns out that the cubic early TMNs are not hugely sensitive to $\sigma$, but in some cases, e.g., $\mathrm{ZrN}$ or $\mathrm{NbN}$, the elastic constant values change by up to $5 \%$ when sigma is increased from $0.02 \mathrm{eV}$ to $0.8 \mathrm{eV}$. Nevertheless, these variations typically take place only for small values of $\sigma$, and a converged behavior is obtained for $\sigma \approx 0.6 \mathrm{eV}$.

The single-crystal elastic constants are summarized in Table IV. When possible to evaluate, we give the $C_{i j} \mathrm{~s}$ based on all three methods described here (i.e., orthorhombic + monoclinic, tetragonal + trigonal, and $A 1+A 6$ deformation modes). Since the $A 1+A 6$ deformation modes were the only ones to provide well converged results for all ten binary nitrides, we show them in Fig. 2 and we will use them in the following analysis for consistency.

A comparison with previous DFT-GGA literature data $^{21,22,41,42,44,45,50}$ yields, apart from a few exceptions, a good agreement with our results. The local density approximation (LDA) based $C_{11}$ elastic constants from the literature ${ }^{20,43,48}$ are higher than our GGA-based data. This is a consequence of over and underbinding of LDA and GGA, respectively, resulting in too small lattice constants and consequently too hard elastic constants in LDA. Finally, although many of the here calculated elastic constants agree well with the available experimental data, in a few cases, the discrepancy is as large as $20 \%$ (e.g., $C_{11}$ of $\left.\mathrm{VN}\right)$.

In all cases, the obtained elastic constants fulfill the stability criteria for cubic crystals,

$$
C_{44}>0, \quad C_{11}>\left|C_{12}\right|, \quad C_{11}+2 C_{12}>0 .
$$



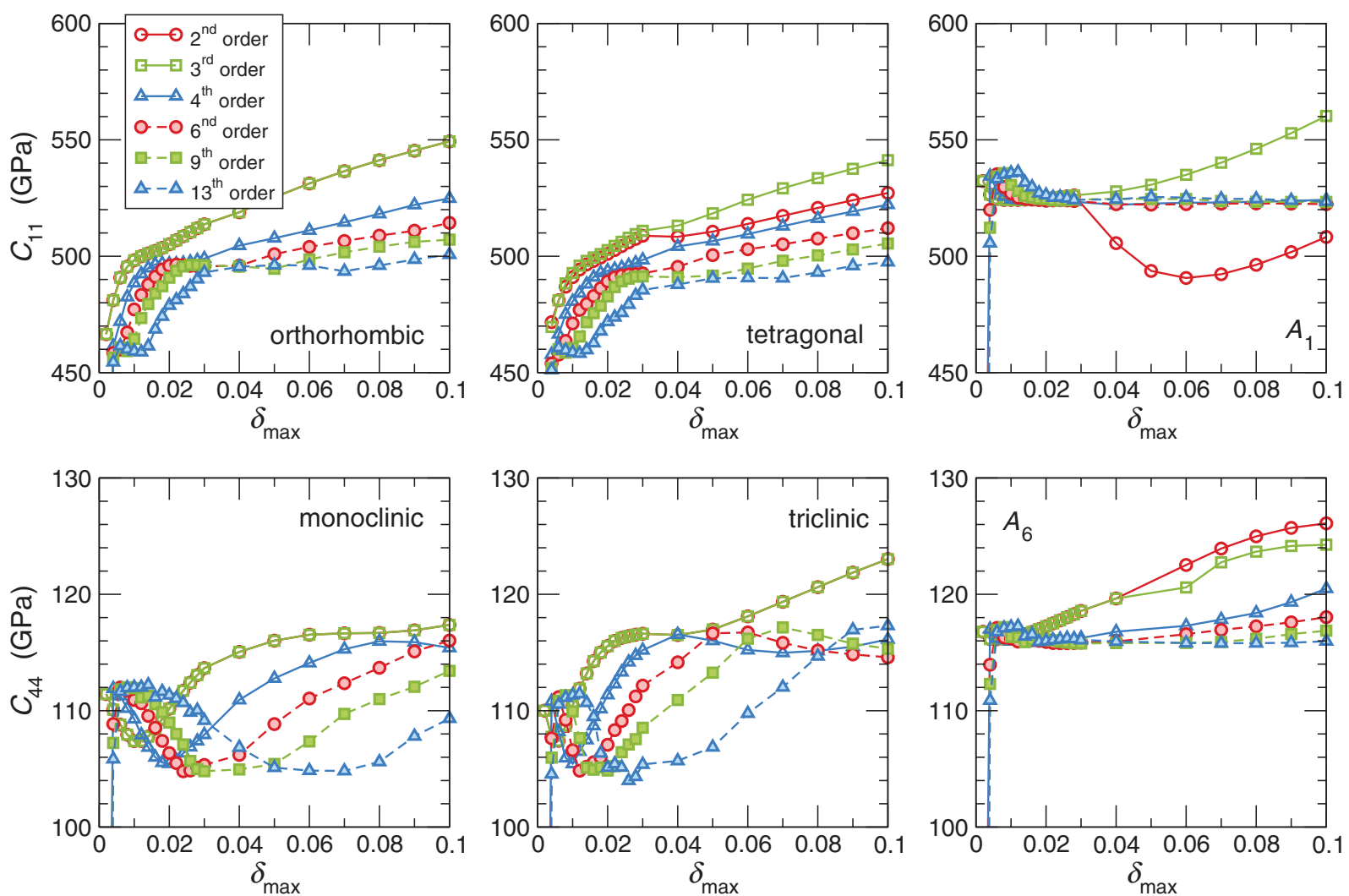

FIG. 1. (Color online) Dependence of the $\mathrm{ZrN}$ elastic constants on the range $-\delta_{\max } \leqslant \delta \leqslant \delta_{\max }$ of deformation taken into account, and the order of the fitting polynomial. The upper and lower rows correspond to $C_{11}$ and $C_{44}$, respectively, as obtained from various approaches described in Sec. II A.

The elastic constant $C_{11}$ is significantly stiffer than the other two elastic constants. Within each row, the $C_{11}$ and $C_{12}$ elastic constants monotonically increase with increasing atomic

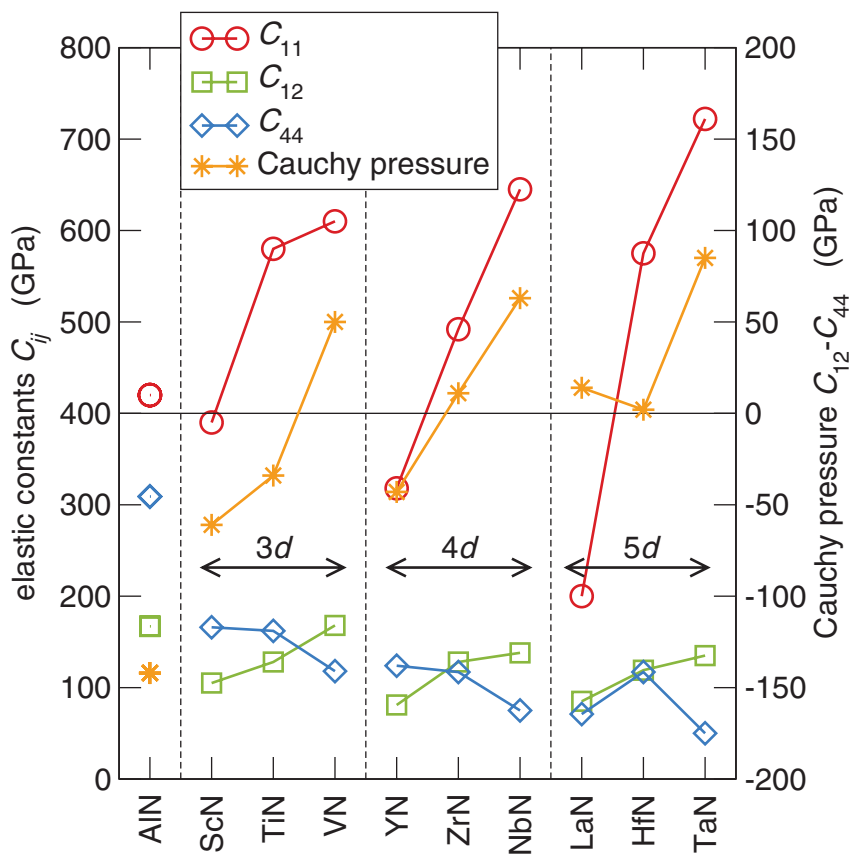

FIG. 2. (Color online) Single-crystal elastic constants $C_{11}, C_{12}$, and $C_{44}$ and resulting Cauchy pressure, $C_{12}-C_{44}$, of the compounds investigated here. number at the same time, $C_{11}$ decreases from Sc to $\mathrm{Y}$ to $\mathrm{La}$ (isovalent IIIB group), while it increases from $\mathrm{V}$ to $\mathrm{Nb}$ to $\mathrm{Ta}$ (isovalent VB group). It has been suggested in the literature ${ }^{51,52}$ that a negative Cauchy pressure $C_{12}-C_{44}<0$ corresponds to more directional bonding, while positive values indicate predominant metallic bonding. Indeed, the calculated Cauchy pressure is most negative for AlN in which a significantly larger charge transfer from cation to anion takes place as, e.g., in TiN. ${ }^{53}$ Cauchy pressure increases to positive values with increasing number of valence electrons within each periodic table row, as those contribute mainly to the metal-metal $d$ - $d$ interactions. ${ }^{19,54}$ These trends may be used in the materials selection process to realize specific requirements.

\section{Directional Young's modulus}

The Young's modulus $E$ in a certain direction $\xi$ is defined as the ratio of longitudinal stress to longitudinal strain in this direction. The elastic compliances $S_{i j}$ are in the case of cubic crystals the solution of the following set of equations: ${ }^{.5}$

$$
\begin{aligned}
& C_{11}=\frac{S_{11}+S_{12}}{\left(S_{11}-S_{12}\right)\left(S_{11}+2 S_{12}\right)}, \\
& C_{12}=\frac{-S_{12}}{\left(S_{11}-S_{12}\right)\left(S_{11}+2 S_{12}\right)}, \\
& C_{44}=\frac{1}{S_{44}} .
\end{aligned}
$$


TABLE IV. Single-crystal elastic constants. "o," "t," and "A" stand for values calculated by orthorhombic and monoclinic, tetragonal, and trigonal, and using the $A 1$ and $A 6$ deformation modes, respectively. "c" and "e" stand for calculated and experimental data from the literature, respectively. The results in bold are used for further analysis of polycrystalline elastic properties.

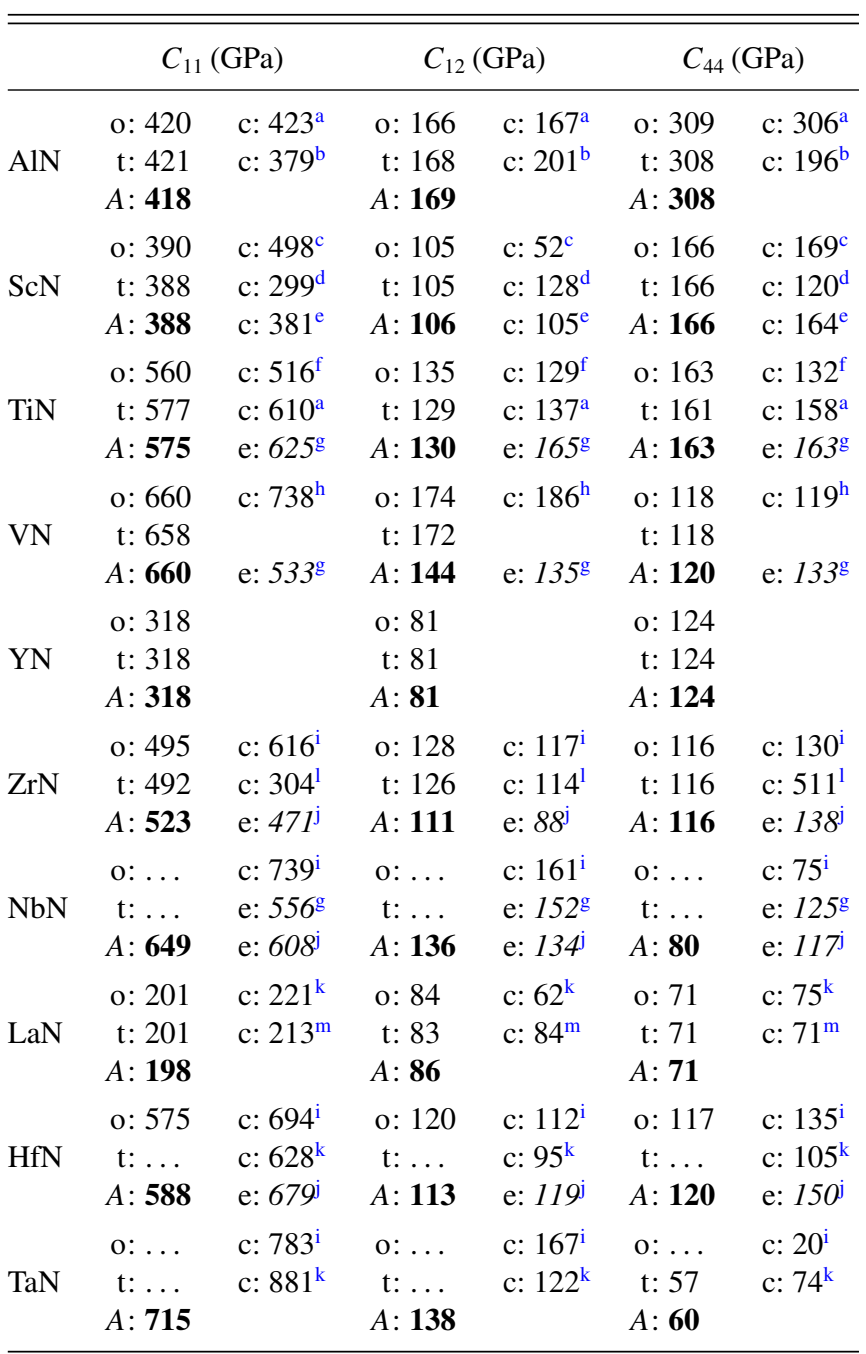

\footnotetext{
${ }^{a}$ Reference 41, GGA.

${ }^{b}$ Reference 42, GGA.

${ }^{c}$ Reference 43, LDA.

${ }^{\mathrm{d}}$ Reference 44, GGA.

${ }^{\mathrm{e}}$ Reference 45, GGA.

${ }^{f}$ Reference 46, LDA.

${ }^{\mathrm{g}}$ Reference 47, exp.

${ }^{\mathrm{h}}$ Reference 48, LDA.

${ }^{\mathrm{i}}$ Reference 20, LDA.

${ }^{\mathrm{j}}$ Reference 49, exp.

${ }^{\mathrm{k}}$ Reference 21, GGA.

${ }^{1}$ Reference 22, GGA.

${ }^{\mathrm{m}}$ Reference 50, GGA.
}

For a cubic crystal, $E_{\xi}$ then reads ${ }^{55}$

$$
\frac{1}{E_{\xi}}=S_{11}-2\left(S_{11}-S_{12}-\frac{1}{2} S_{44}\right)\left(l_{1}^{2} l_{2}^{2}+l_{2}^{2} l_{3}^{2}+l_{1}^{2} l_{3}^{2}\right),
$$

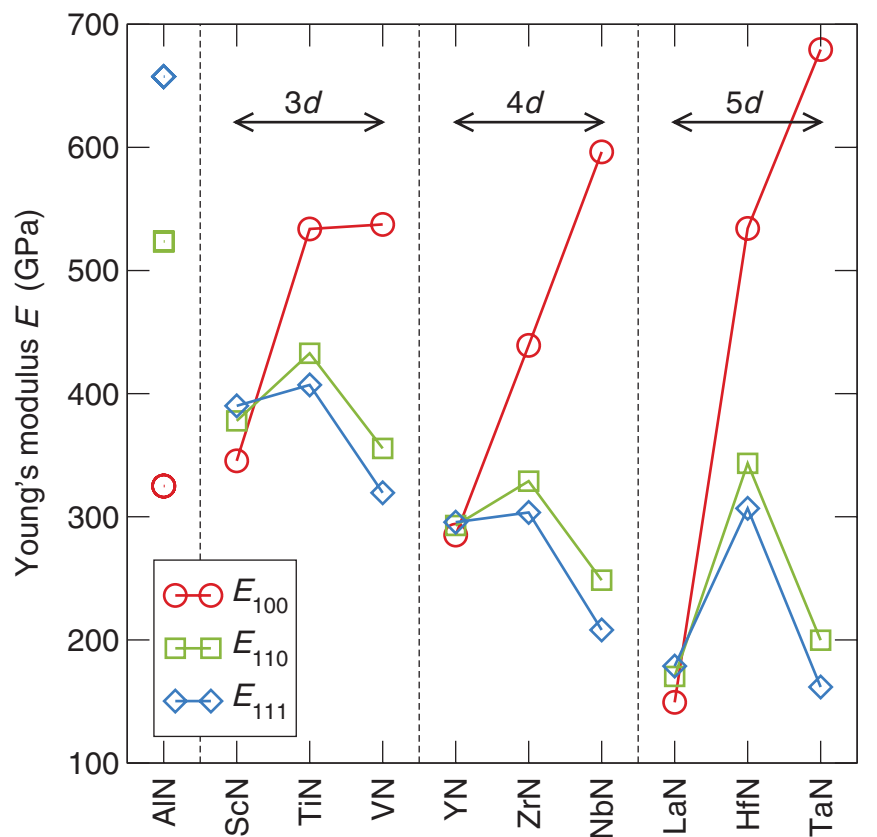

FIG. 3. (Color online) Young's modulus in the $\langle 100\rangle,\langle 110\rangle$, and $\langle 111\rangle$ directions.

where $l_{1}, l_{2}$, and $l_{3}$ are the directional cosines of $\xi$. For $\langle 100\rangle$, $\langle 110\rangle$, and $\langle 111\rangle$ directions, this becomes

$$
\begin{aligned}
& E_{\langle 100\rangle}=1 / S_{11}, \\
& E_{\langle 110\rangle}=1 /\left[S_{11}-\frac{1}{2}\left(S_{11}-S_{12}-\frac{1}{2} S_{44}\right)\right], \\
& E_{\langle 111\rangle}=1 /\left[S_{11}-\frac{2}{3}\left(S_{11}-S_{12}-\frac{1}{2} S_{44}\right)\right] .
\end{aligned}
$$

The results are plotted in Fig. 3. The Young's modulus in $\langle 100\rangle$ follows mostly the same trend as $C_{11}$, since the $C_{11}$ elastic constant has the strongest contribution to $E_{\langle 100\rangle}$. There is a considerable difference between the semiconducting compounds AlN, ScN, YN, and $\mathrm{LaN}$, in which the $\langle 100\rangle$ direction becomes the softest, and the metallic TiN, $\mathrm{VN}, \mathrm{ZrN}$, $\mathrm{NbN}, \mathrm{HfN}$, and TaN, where the $\langle 100\rangle$ direction is clearly the strongest. In addition, the Young's modulus of AlN in the $\langle 111\rangle$ direction is more than 1.5 times larger than in any other of the here investigated TMNs. This is mainly caused by the high value of $C_{44}$ of AlN, suggesting that AlN is much stronger in shear deformation than the other TMN.

To quantify the anisotropy, we employ the Zener's anisotropy ratio, ${ }^{56} \mathrm{~A}$, defined as

$$
A=\frac{2 C_{44}}{C_{11}-C_{12}} .
$$

The results, together with the ratio $E_{\langle 111\rangle} / E_{\langle 100\rangle}$, which provides similar information, are shown in Fig. 4 . The results suggest that AlN is clearly stiffer in the $\langle 111\rangle$ than in the $\langle 100\rangle$ direction. The opposite result is obtained for the group IVB and VB TMN where the $\langle 100\rangle$ direction is the stiffest. The group IIIB semiconducting TMN exhibit values of both, $A$ and $E_{\langle 111\rangle} / E_{\langle 100\rangle}$ very close to 1 . This implies that their elastic behavior is almost isotropic. The most isotropic response is predicted for $\mathrm{YN}$ with $A=1.05$. The (an)isotropy of 


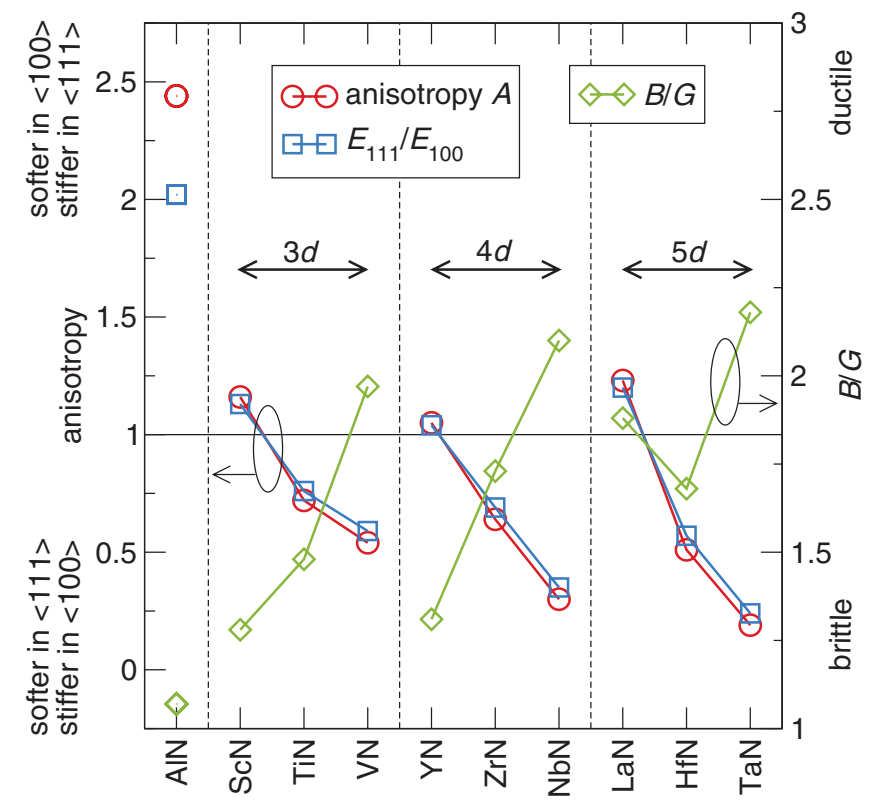

FIG. 4. (Color online) Zener's anisotropy ratio $E_{\langle 111\rangle} / E_{\langle 100\rangle}$ and $B / G$ values for the ten nitrides studied here.

the Young's modulus is visualized in Fig. 5. Figure 5(b) demonstrates the isotropic elastic response of $\mathrm{YN}$, while the comparison of Figs. 5(a) and 5(c) shows the qualitatively different elastic response of AlN and TiN.

Some insight into these trends can be gained from considering the differences in bonding. The bonds in AlN are strongly ionic, ${ }^{53}$ while the TMNs contain a significant part of the covalent bonding. ${ }^{19}$ Since the covalent bond is stronger than the ionic, this can rationalize why AIN has the smallest value of $E_{\langle 100\rangle}$. When going from group IIIB to VB elements within each row, the extra electrons fill the bonding metal-metal orbitals, while the hybridized $s p^{3} d^{2}$ states move to lower energies. ${ }^{19,54}$ This can be interpreted as strengthening of the hybridized $s p^{3} d^{2}$ bonds that are oriented along the $\langle 100\rangle$ directions. As for the high $C_{44}$ elastic constant of AlN, one may argue that since there are no $d$ electrons available to form the metal-metal bonds in the $\langle 110\rangle$ directions (as it is the case for the IVB and VB group elements), upon a shear deformation, a significantly increased repulsion between $\mathrm{Al}-\mathrm{Al}$ and N-N ions as they get closer occurs, which causes the high value of $E_{\langle 111\rangle}$.

\section{Polycrystalline properties}

Several models exist that assess the isotropic polycrystalline elastic properties using the anisotropic single-crystal elastic
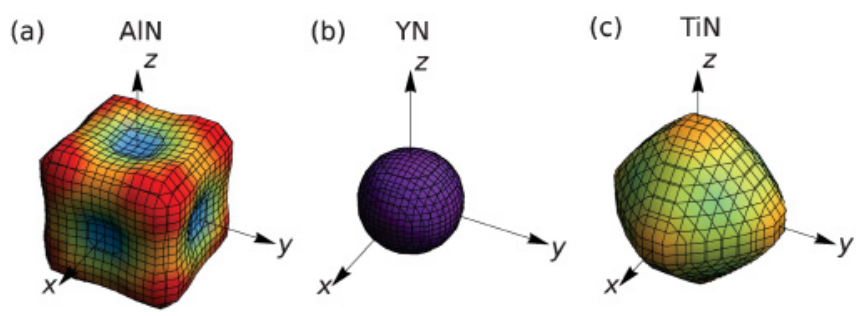

FIG. 5. (Color online) 3D representation of the directional dependence of Young's modulus for (a) AlN, (b) YN, and (c) TiN. constants of a given material. Voigt's approach ${ }^{57}$ of constant strains in all grains yields the upper limit, $G_{V}$ and $E_{V}$, to the polycrystalline shear and Young's moduli, respectively. On the other hand, Reuss ${ }^{58}$ proposed to apply constant stresses in all grains, which yields lower limits $G_{R}$ and $E_{R}$. Taking $B_{V}=B_{R}=B$, where the bulk modulus $B$ is obtained from the Birch-Murnaghan equation of state, ${ }^{24}$ one gets

$$
\begin{gathered}
G_{V}=\frac{C_{11}-C_{12}+3 C_{44}}{5}, \\
G_{R}=\frac{5}{4\left(S_{11}-S_{12}\right)+3 S_{44}}, \\
E_{\alpha}=\frac{9 B G_{\alpha}}{3 B+G_{\alpha}}, \quad \alpha=V, R .
\end{gathered}
$$

Finally, Hershey ${ }^{59}$ derived an equation for self-consistently calculating the shear modulus, $G_{H}$. In this approach, $G_{H}$ is the real positive root of the following fourth-order polynomial:

$$
\begin{aligned}
64 G_{H}^{4}+16\left(4 C_{11}+5 C_{12}\right) G_{H}^{3} \\
\quad+\left(3\left(C_{11}+2 C_{12}\right)\left(5 C_{11}+4 C_{12}\right)\right. \\
\left.\quad-8\left(7 C_{11}-4 C_{12}\right) C_{44}\right) G_{H}^{2} \\
\quad-\left(29 C_{11}-20 C_{12}\right)\left(C_{11}+2 C_{12}\right) C_{44} G_{H} \\
\quad-3\left(C_{11}+2 C_{12}\right)^{2}\left(C_{11}-C_{12}\right) C_{44}=0 .
\end{aligned}
$$

This equation can be simplified by dividing it with $\left(3 C_{11}+\right.$ $\left.6 C_{12}+8 G\right)$ to a third-order polynomial ${ }^{60}$ with the same positive real root:

$$
\begin{aligned}
8 G^{3} & +\left(5 C_{11}+4 C_{12}\right) G^{2}-C_{44}\left(7 C_{11}-4 C_{12}\right) G \\
& -C_{44}\left(C_{11}-C_{12}\right)\left(C_{11}+2 C_{12}\right)=0 .
\end{aligned}
$$

Subsequently, Eq. (20) is used to estimate the Young's modulus within Hershey's approach.

The calculated polycrystalline elastic constants are shown in Fig. 6 together with the bulk modulus. They fit well with the few accessible experimental data points included (black full symbols). The trends in $E$ and $G$ are akin: the maximum value in each row of the periodic table is obtained for the group IVB TMNs. The spread between $G_{R}$ and $G_{V}$ (shaded in Fig. 6), as well as between $E_{R}$ and $E_{V}$ is very small for the group IIIB and IVB TMNs, suggesting that the elastic properties of polycrystals of these materials will not be hugely influenced by the misorientations of individual grains. A different situation is obtained for AlN and group VB TMNs (in particular, for $\mathrm{NbN}$ and $\mathrm{TaN}$ ), where the Reuss-Voigt range is quite large. As shown in Ref. 65, the ratio between the Voigt and Reuss bounds depends nonlinearly on the anisotropy factor $A$. The ratio becomes particularly large when the anisotropy $A$ approaches 0 , as in the case of $\mathrm{NbN}$ and TaN. As a consequence, these materials are expected to be strongly affected by the actual microstructures (i.e., not only by the grain orientations, but also by the shape of the grain).

Based on an evaluation of a large experimental data set, Pugh $^{66}$ proposed that the higher (lower) the $B / G$ ratio is the more ductile (brittle) the material is. This ratio is plotted in Fig. 4. In general, the ductility increases from IIIB to VB group (e.g., with increasing number of valence electrons and thus increasing amount of metallic bonding), and within each group from lighter to heavier elements. 


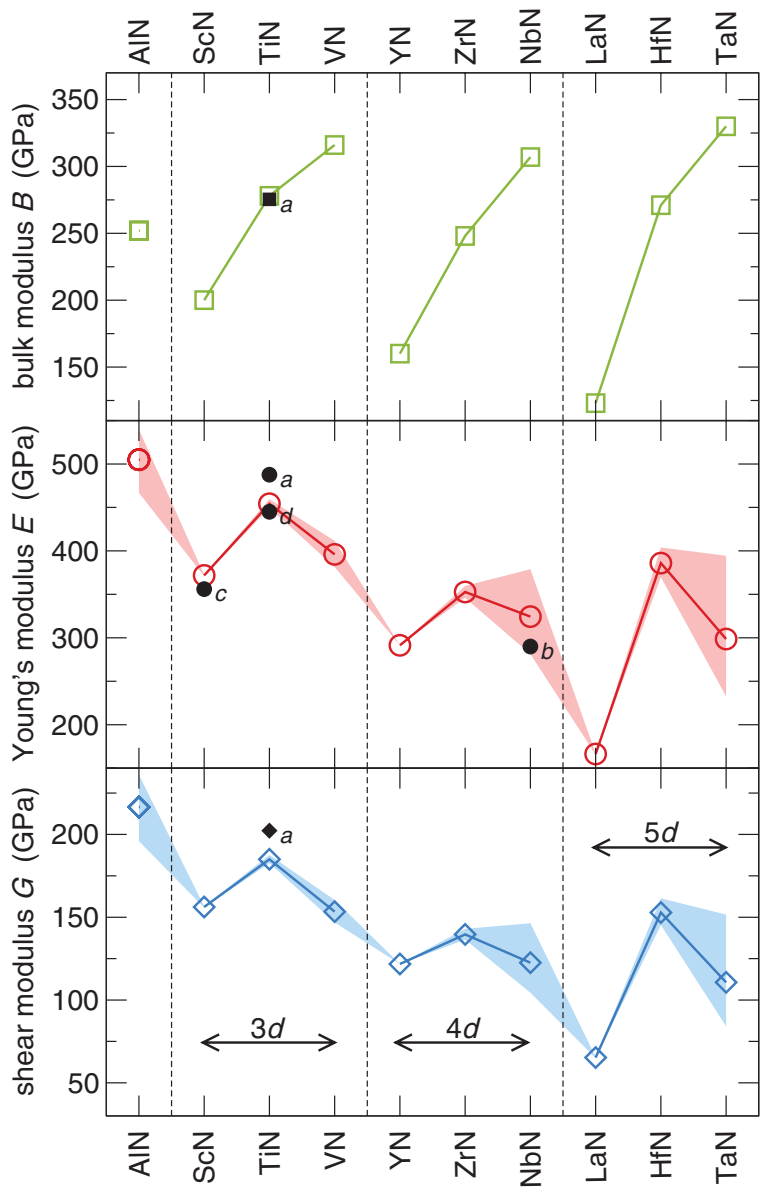

FIG. 6. (Color online) Calculated bulk modulus $B$, polycrystalline Young's $E$, and shear $G$ moduli. The shaded area corresponds to the Reuss's and Voigt's limit cases. The full symbols denote experimental values from literature. ( $\left({ }^{\mathrm{a}}\right.$ Reference $61,{ }^{\mathrm{b}}$ Reference 62 , ${ }^{\mathrm{c}}$ Reference 63, ${ }^{\mathrm{d}}$ Reference 64.)

\section{E. Third-order elastic constants}

The methodology employing the deformation matrices A1$A 6$ allows also to easily estimate third-order elastic constants (TOECs), by following Eq. (12) and relations in Table I. TOECs $C_{i j k}$ appear in the Taylor series expansion of the strain energy

$$
\begin{aligned}
U & =\frac{1}{2} \sum_{i j} C_{i j} \eta_{i} \eta_{j}+\frac{1}{6} \sum_{i j k} C_{i j k} \eta_{i} \eta_{j} \eta_{k}+\ldots \\
& =\frac{1}{2} \sum_{i j}\left(C_{i j}+\frac{1}{3} \sum_{k} C_{i j k} \eta_{k}+\ldots\right) \eta_{i} \eta_{j},
\end{aligned}
$$

where $\varepsilon_{\alpha \beta}=\left(1+\delta_{\alpha \beta}\right) \eta_{i} / 2$ is the relationship between components $\varepsilon_{\alpha \beta}$ of the Lagrangian strain tensor and six components $\eta_{i}$ of a corresponding vector in Voigt notation. ${ }^{25,26,60}$ According to the above equation, TOECs give corrections when applying such large strains that linear elasticity no longer applies. TOECs are thus useful to describe the pressure dependence of second-order elastic constants $C_{i j}$ or thermal properties of solids. ${ }^{67}$ This can be of a particular interest for thin films where residual stresses in the range of several GPa can be realized.
TABLE V. Third-order elastic constants as obtained from the six deformation modes $A 1-A 6$ [Eq. (12)]

\begin{tabular}{lrrrrrr}
\hline \hline & $\begin{array}{c}C_{111} \\
(\mathrm{GPa})\end{array}$ & $\begin{array}{c}C_{112} \\
(\mathrm{GPa})\end{array}$ & $\begin{array}{c}C_{123} \\
(\mathrm{GPa})\end{array}$ & $\begin{array}{c}C_{144} \\
(\mathrm{GPa})\end{array}$ & $\begin{array}{c}C_{166} \\
(\mathrm{GPa})\end{array}$ & $\begin{array}{r}C_{456} \\
(\mathrm{GPa})\end{array}$ \\
\hline $\mathrm{AlN}$ & -5200 & -400 & 330 & 320 & -850 & 280 \\
$\mathrm{ScN}$ & -5100 & -190 & 260 & 200 & -330 & 215 \\
TiN & -7100 & -370 & 430 & 175 & -475 & 60 \\
$\mathrm{VN}$ & -8000 & -400 & 420 & 541 & -450 & -235 \\
YN & -4100 & -160 & 180 & 180 & -225 & 185 \\
$\mathrm{ZrN}$ & -6450 & -310 & 370 & 150 & -370 & -5 \\
NbN & -8600 & -190 & 115 & 300 & -480 & -180 \\
LaN & -1200 & -550 & 650 & 140 & -80 & 135 \\
HfN & -7050 & -350 & 520 & 170 & -450 & -220 \\
TaN & -9800 & -20 & -190 & 340 & -600 & -230 \\
\hline
\end{tabular}

The computed TOECs for the binary systems investigated in this work are summarized in Table V. For isotropic aggregates of cubic crystals, Lubarda ${ }^{60}$ derived equations for Voigt- and Reuss-type averages of TOECs. These equations are equivalents to the elastic constants expressed by Eqs. (18)-(20). The corresponding formulas are briefly summarized in Appendix. The three polycrystalline Voigt- and Reuss-type TOECs $C_{123}, C_{144}$, and $C_{456}$ are presented in Fig. 7 as the upper and lower boundaries of the shaded areas. These boundaries provide an estimate for the expected spread of the data and

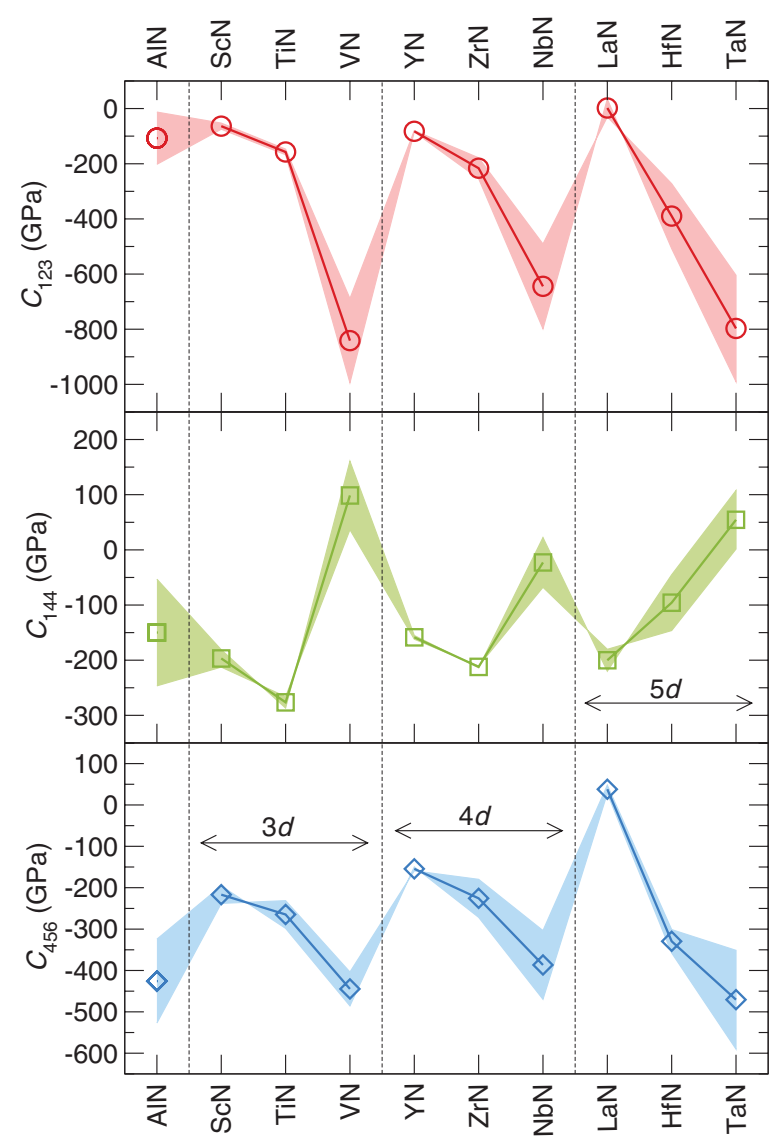

FIG. 7. (Color online) The polycrystalline third-order elastic constants. The shaded area corresponds, for each compound, to the spread between Voigt- and Reuss-type approach, while the solid line with data points represents Hill's average [see Eq. (24)]. 
depend on the actual microstructure. The lines in Fig. 7 represent the Hill's average

$$
C_{i j k, \mathrm{H}}=\frac{C_{i j k, \mathrm{~V}}+C_{i j k, \mathrm{R}}}{2}
$$

of the Voigt-type $C_{i j k, \mathrm{~V}}$ and Reuss-type $C_{i j k, \mathrm{R}}$ TOECs. The six TOECs describing a crystal with the cubic symmetry are in the isotropic case related by

$$
\begin{gathered}
C_{111}=C_{123}+6 C_{144}+8 C_{456}, \\
C_{112}=C_{123}+C_{244}, \\
C_{166}=C_{144}+2 C_{456} .
\end{gathered}
$$

The results suggest that in each row of the periodic table, $C_{123}$ and $C_{456}$ decrease to more negative values with increasing number of valence electrons from 3 to 5 . For $C_{144}$, no clear trend is observed. Since the TOECs are mostly negative, second-order elastic constants get stiffer with compressive stresses and they soften under tension [compare with Eq. (23)].

\section{CONCLUSIONS}

Calculating elastic properties using density functional theory is a powerful technique, in particular when material phases of single crystals are not experimentally accessible. In this paper we provided a coherent description of the elastic behavior of nine binary early transition-metal nitrides $(\mathrm{ScN}$, TiN, VN, YN, ZrN, NbN, LaN, HfN, and TaN) and AlN. These binary compounds are of high technological interest for designing materials with application-tailored properties. Single-crystal elastic constants $C_{i j}$ and directionally resolved Young's moduli $E$ in $\langle 100\rangle,\langle 110\rangle$, and $\langle 111\rangle$ directions are provided. The results clearly indicate the special position of AlN. This material has the largest Young's modulus $E$ along $\langle 111\rangle$, while all group IVB and VB nitrides exhibit the largest $E$ value along $\langle 100\rangle$. These trends could be rationalized by analyzing the bonding characteristics of these compounds. Computing the elastic anisotropy we find that YN followed by $\mathrm{ScN}$ and $\mathrm{LaN}$ are the materials closest to the elastically isotropic behavior. Finally, the polycrystalline elastic properties (Young's and shear modulus) were calculated. Good agreement with the rather scarce available experimental data was obtained.

\section{ACKNOWLEDGMENT}

The authors greatly acknowledge the financial support by the START Program (Y371) of the Austrian Science Fund (FWF).

\section{APPENDIX: EQUATIONS FOR THE POLYCRYSTALLINE TOECS}

A detailed derivation of the equations below was given by Lubarda. ${ }^{60}$ Here, we only summarize the final results for the reader's perusal.

The Voigt-type TOECs $C_{i j k, \mathrm{~V}}$ are obtained from the singlecrystal elastic constants $C_{i j k}$ as

$$
\begin{aligned}
C_{123, \mathrm{v}}= & \frac{1}{35}\left(C_{111}+18 C_{112}+16 C_{123}\right. \\
& \left.-30 C_{144}-12 C_{166}+16 C_{456}\right), \\
C_{144, \mathrm{~V}}= & \frac{1}{35}\left(C_{111}+4 C_{112}-5 C_{123}\right. \\
& \left.+19 C_{144}+2 C_{166}-12 C_{456}\right), \\
C_{456, \mathrm{v}}= & \frac{1}{35}\left(C_{111}-3 C_{112}+2 C_{123}\right. \\
& \left.-9 C_{144}+9 C_{166}+9 C_{456}\right) .
\end{aligned}
$$

The Reuss-type estimates of TEOCs $C_{i j k, \mathrm{R}}$ can be calculated as

$$
\begin{aligned}
C_{456, \mathrm{R}}= & \frac{1}{35}\left(\frac{5 A}{2 A+3}\right)^{3}\left[C_{111}-3 C_{112}+2 C_{123}\right. \\
& \left.-\frac{9}{A^{2}}\left(C_{144}-C_{166}\right)+\frac{9}{A^{3}} C_{456}\right] \\
C_{144, \mathrm{R}}= & \frac{1}{3}\left[\frac{A}{2 A+3}\left[C_{111}-C_{123}+\frac{3}{A}\left(C_{144}+2 C_{166}\right)\right]\right. \\
- & \left.4 C_{456, \mathrm{R}}\right] \\
9 & C_{123, \mathrm{R}}+18 C_{144, \mathrm{R}}+8 C_{456, \mathrm{R}} \\
= & 9 C_{123, \mathrm{~V}}+18 C_{144, \mathrm{~V}}+8 C_{456, \mathrm{~V}}
\end{aligned}
$$

where $A$ is the anisotropy ratio given by Eq. (17). It can be seen that in case of an isotropic materials $(A=1)$, the two approaches give the same results [compare, e.g., Eqs. (A3) and (A4)].

\footnotetext{
*david.holec@unileoben.ac.at

${ }^{1}$ S. PalDey and S. C. Deevi, Mater. Sci. Eng. A 342, 58 (2003).

${ }^{2}$ P. H. Mayrhofer, C. Mitterer, L. Hultman, and H. Clemens, Prog. Mater. Sci. 51, 1032 (2006).

${ }^{3}$ S. C. Jain, M. Willander, J. Narayan, and R. V. Overstraeten, J. Appl. Phys. 87, 965 (2000).

${ }^{4}$ P. Chen, Z. Xiong, J. Luo, J. Lin, and K. L. Tan, (London) 420, 302 (2002).

${ }^{5}$ P. H. Mayrhofer, A. Hörling, L. Karlsson, J. Sjolen, T. Larsson, C. Mitterer, and L. Hultman, Appl. Phys. Lett. 83, 2049 (2003).

${ }^{6}$ M. Moser and P. H. Mayrhofer, Scr. Mater. 57, 357 (2007).

${ }^{7}$ P. H. Mayrhofer, R. Rachbauer, and D. Holec, Scr. Mater. 63, 807 (2010).
}

${ }^{8}$ L. Chen, D. Holec, Y. Du, and P. H. Mayrhofer, Thin Solid Films 519, 5503 (2011).

${ }^{9}$ F. Rovere, D. Music, J. Schneider, and P. H. Mayrhofer, Acta Mater. 58, 2708 (2010).

${ }^{10}$ H. Holleck, M. Lahres, and P. Woll, Surf. Coat. Technol. 41, 179 (1990).

${ }^{11}$ T. Friesen, J. Haupt, W. Gissler, A. Barna, and P. B. Barna, Surf. Coat. Technol. 48, 169 (1991).

${ }^{12}$ J. Paulitsch, M. Schenkel, A. Schintlmeister, H. Hutter, and P. H. Mayrhofer, Thin Solid Films 518, 5553 (2010).

${ }^{13}$ J. Mackerle, Modell. Simul. Mater. Sci. Eng. 13, 935 (2005).

${ }^{14}$ K. Holmberg, A. Matthews, and H. Ronkainen, Tribol. Int. 31, 107 (1998). 
${ }^{15}$ C. Walter and C. Mitterer, Surf. Coat. Technol. 203, 3286 (2009).

${ }^{16}$ J. Häglund, G. Grimvall, T. Jarlborg, and A. F. Guillermet, Phys. Rev. B 43, 14400 (1991).

${ }^{17}$ J. Häglund, A. Fernández Guillermet, G. Grimvall, and M. Körling, Phys. Rev. B 48, 11685 (1993).

${ }^{18}$ B. Eck, R. Dronskowski, M. Takahashi, and S. Kikkawa, J. Mater. Chem. 9, 1527 (1999).

${ }^{19}$ C. Stampfl, W. Mannstadt, R. Asahi, and A. J. Freeman, Phys. Rev. B 63, 155106 (2001).

${ }^{20}$ Z. Wu, X.-J. Chen, V. V. Struzhkin, and R. E. Cohen, Phys. Rev. B 71, 214103 (2005).

${ }^{21}$ E. Zhao and Z. Wu, J. Solid State Chem. 181, 2814 (2008).

${ }^{22}$ D. Cheng, S. Wang, and H. Ye, J. Alloys Compd. 377, 221 (2004).

${ }^{23}$ We deliberately leave our $\mathrm{CrN}$ for this report since its magnetic configuration deserves a deeper analysis, see, e.g., Refs. 68-70.

${ }^{24}$ F. Birch, Phys. Rev. 71, 809 (1947).

${ }^{25}$ J. Zhao, J. M. Winey, and Y. M. Gupta, Phys. Rev. B 75, 094105 (2007).

${ }^{26}$ M. Łopuszyński and J. A. Majewski, Phys. Rev. B 76, 045202 (2007).

${ }^{27}$ There are in total six independent third-order elastic constants for materials with the cubic symmetry.

${ }^{28}$ P. Hohenberg and W. Kohn, Phys. Rev. 136, B864 (1964).

${ }^{29}$ W. Kohn and L. J. Sham, Phys. Rev. 140, A1133 (1965).

${ }^{30}$ G. Kresse and J. Hafner, Phys. Rev. B 47, 558 (1993).

${ }^{31}$ G. Kresse and J. Furthmüller, Phys. Rev. B 54, 11169 (1996).

${ }^{32}$ G. Kresse and D. Joubert, Phys. Rev. B 59, 1758 (1999).

${ }^{33}$ Y. Wang and J. P. Perdew, Phys. Rev. B 44, 13298 (1991).

${ }^{34}$ A. Fernández Guillermet, J. Häglund, and G. Grimvall, Phys. Rev. B 45, 11557 (1992).

${ }^{35}$ F. Rovere, D. Music, S. Ershov, M. Baben, H.-G. Fuss, P. H. Mayrhofer, and J. M. Schneider, J. Phys. D: Appl. Phys. 43, 035302 (2010b).

${ }^{36}$ Powder diffraction files 00-046-1200 (AlN), 00-045-0978 ( $\left.\mathrm{ScN}\right)$, 03-065-0565 (TiN), 00-035-0768 (VN), 00-035-0779 (YN), 00035-0753 (ZrN), 03-065-5011 (NbN), 03-065-9339 (LaN), 00-0330592 (HfN), 03-065-9404 (TaN), International center for diffraction data, PDF-2/release 2007 (2007).

${ }^{37}$ R. Rachbauer, D. Holec, and P. H. Mayrhofer, Appl. Phys. Lett. 97, 151901 (2010).

${ }^{38}$ CRC Handbook of Chemistry and Physics, 85th ed., edited by D. Lide (CRC Press, 2004).

${ }^{39}$ H. O. Pierson, in Handbook of Refractory Carbides and Nitrides: Properties, Characteristics, Processing, and Applications (William Andrew, 1996), p. 362.

${ }^{40}$ A. Udyansky, J. von Pezold, A. Dick, and J. Neugebauer, Phys. Rev. B 83, 184112 (2011).

${ }^{41}$ A. Wang, S. Shang, Y. Du, Y. Kong, L. Zhang, L. Chen, D. Zhao, and Z. Liu, Comput. Mater. Sci. 48, 705 (2010).

${ }^{42}$ S. Saib and N. Bouarissa, J. Phys. Chem. Solids 67, 1888 (2006).

${ }^{43}$ P. Pandit, B. Rakshit, and S. P. Sanyal, Phys. Status Solidi B 248, 921 (2011).
${ }^{44}$ Y. Oussaifi, A. B. Fredj, M. Debbichi, N. Bouarissa, and M. Said, Semicond. Sci. Technol. 22, 641 (2007).

${ }^{45}$ W. Feng, S. Cui, H. Hu, G. Zhang, Z. Lv, and Z. Gong, Physica B 405, 2599 (2010).

${ }^{46}$ M. Zhang and J. He, Surf. Coat. Technol. 142-144, 125 (2001).

${ }^{47}$ J. O. Kim, J. D. Achenbach, P. B. Mirkarimi, M. Shinn, and S. A. Barnett, J. Appl. Phys. 72, 1805 (1992).

${ }^{48}$ W. Wolf, R. Podloucky, T. Antretter, and F. D. Fischer, Philos. Mag. B 79, 839 (1999).

${ }^{49}$ X.-J. Chen, V. V. Struzhkin, Z. Wu, M. Somayazulu, J. Qian, S. Kung, A. N. Christensen, Y. Zhao, R. E. Cohen, H.-k. Mao, and R. J. Hemley, Proc. Natl. Acad. Sci. USA 102, 3198 (2005).

${ }^{50}$ Y. Ciftci, K. Çolakoglu, E. Deligoz, and H. Ozisik, Mater. Chem. Phys. 108, 120 (2008).

${ }^{51}$ K. Chen, L. R. Zhao, and J. S. Tse, J. Appl. Phys. 93, 2414 (2003).

${ }^{52}$ D. G. Pettifor, Mater. Sci. Technol. 8, 345 (1992).

${ }^{53}$ D. Holec, R. Rachbauer, D. Kiener, P. D. Cherns, P. M. F. J. Costa, C. McAleese, P. H. Mayrhofer, and C. J. Humphreys, Phys. Rev. B 83, 165122 (2011).

${ }^{54}$ R. Rachbauer, D. Holec, M. Lattemann, L. Hultman, and P. H. Mayrhofer, Int. J. Mater. Res. (formerly Zeitschrift fuer Metallkunde) 102, 735 (2011).

${ }^{55}$ J. Nye, Physical Properties of Crystals, 1st ed. (Clarendon, Oxford, 1957).

${ }^{56}$ S. I. Ranganathan and M. Ostoja-Starzewski, Phys. Rev. Lett. 101, 055504 (2008).

${ }^{57}$ W. Voigt, Lehrbuch der Kristallphysik (B. B. Teubner, Leipzig, 1928).

${ }^{58}$ A. Reuss, Z. Angew. Math. Mech. 9, 49 (1929).

${ }^{59}$ A. V. Hershey, J. Appl. Mech. 21, 236 (1954).

${ }^{60}$ V. A. Lubarda, J. Mech. Phys. Solids 45, 471 (1997).

${ }^{61}$ H. Chen, F. Peng, H.-k. Mao, G. Shen, H.-P. Liermann, Z. Li, and J. Shu, J. Appl. Phys. 107, 113503 (2010).

${ }^{62}$ M. Benkahoul, E. Martinez, A. Karimi, R. Sanjinés, and F. Lévy, Surf. Coat. Technol. 180, 178 (2004).

${ }^{63}$ D. Gall, I. Petrov, N. Hellgren, L. Hultman, J. E. Sundgren, and J. E. Greene, J. Appl. Phys. 84, 6034 (1998).

${ }^{64}$ H. Ljungcrantz, M. Oden, L. Hultman, J. E. Greene, and J.-E. Sundgren, J. Appl. Phys. 80, 6725 (1996).

${ }^{65}$ M. Friák, W. A. Counts, D. Raabe, and J. Neugebauer, Phys. Status Solidi B 245, 2636 (2008).

${ }^{66}$ S. F. Pugh, Philos. Mag. 45, 823 (1954).

${ }^{67}$ T. Çagin and J. R. Ray, Phys. Rev. B 38, 7940 (1988).

${ }^{68}$ B. Alling, T. Marten, and I. A. Abrikosov, Nat. Mater. 9, 283 (2010).

${ }^{69}$ B. Alling, T. Marten, and I. A. Abrikosov, Phys. Rev. B 82, 184430 (2010).

${ }^{70}$ F. Rivadulla, M. Bañobre López, C. X. Quintela, A. Piñeiro, V. Pardo, D. Baldomir, M. A. López-Quintela, J. Rivas, C. A. Ramos, H. Salva, J.-S. Zhou, and J. B. Goodenough, Nat. Mater. 8, 947 (2009). 